\title{
Mediterranean offshore extreme wind analysis from the 44-year HIPOCAS database: different approaches towards the estimation of return periods and levels of extreme values
}

\author{
M. G. Sotillo ${ }^{1}$, R. Aznar ${ }^{2}$, and F. Valero ${ }^{2}$ \\ ${ }^{1}$ Área de Medio Físico, Puertos del Estado (PE), Madrid, Spain \\ ${ }^{2}$ Dpto. Astrofísica y CC. de la Atmósfera, Universidad Complutense de Madrid, Spain \\ Received: 7 October 2005 - Revised: 1 December 2005 - Accepted: 19 December 2005 - Published: 10 April 2006
}

\begin{abstract}
The present contribution addresses the performance of a statistical extreme wind analysis over the whole Mediterranean Basin. Estimations of return periods and levels are obtained over offshore areas through analysis of annual wind maxima. An alternative regional statistic method, based on regional L-moments, is also proposed. This regional technique allows increasing the sample size, using data from a homogeneous region instead of only from a single location, reducing therefore uncertainty. The performed statistical extreme wind analyses provide a detailed assessment of Mediterranean offshore high wind areas.
\end{abstract}

\section{Introduction}

From last decades, the world is seeing a progressive increase of interest on using sustainable and clean renewable energies. In this framework, wind energy is seen today as a proven useful technology. Nowadays, many efforts are leading to use the high potential offshore wind energy resources. It is expected that the use of this offshore resource is likely to grow significantly in the next decade. Taking into account this scenery, the detailed assessment of offshore wind resource arises as a decisive requirement. Part of such assessment is based on extreme offshore wind atlas generated mainly from global reanalysis and satellite data. Whereas satellite data present as a shortcoming its temporal inhomogeneity (due to devices changes), global re-analyses are not completely adequate to assess realistically extreme wind events due to a general underestimation of wind speed related to its coarse spatial resolution. This global reanalysis limitation seems to be decisive over areas such as the Mediterranean Basin, marked by a complex land-sea distribution and significant orography. The HIPOCAS Mediterranean long-term (1958-

Correspondence to: M. G. Sotillo

(marcos@ puertos.es)
2001) wind database comes to overcome the aforementioned re-analysis shortcoming, providing a high resolution longterm Mediterranean wind data set useful to perform extreme wind analysis.

\section{HIPOCAS Mediterranean database}

The long-term 44-year (1958-2001) HIPOCAS database is the result of an atmospheric hindcast performed over the whole Mediterranean Basin, by means of the regional atmospheric model REMO, covering the whole Mediterranean basin by a grid with horizontal resolution of $0.5^{\circ} \times 0.5^{\circ}$. NCEP/NCAR global reanalysis data from the T62 grid, equivalent to a horizontal resolution of about $210 \mathrm{~km}$ (Kalnay et al., 1996) were used as initial and boundary conditions. Further information on the HIPOCAS Mediterranean database and its generation can be found in Sotillo et al. (2005). This work shows a HIPOCAS wind data validation over offshore areas. Another HIPOCAS wind validation, but this time focussed on extreme wind events, is presented in Sotillo et al. (2003). In this work, offshore Mediterranean HIPOCAS extreme wind events were compared with the available in-situ measurements, as well as with the ones reanalyzed by NCEP. These comparisons showed that HIPOCAS data reproduce more efficiently Mediterranean regional winds and enhanced the confidence on such hindcasted database to perform extreme wind analysis.

\section{Extreme wind analysis: standard approach}

Extreme wind analyses were carried out using to this aim the HIPOCAS annual-maxima wind speed data obtained along the whole 44 years over the Mediterranean and the northeastern Atlantic. Such extreme analyses comprised the calculation of return periods and their corresponding quantile 

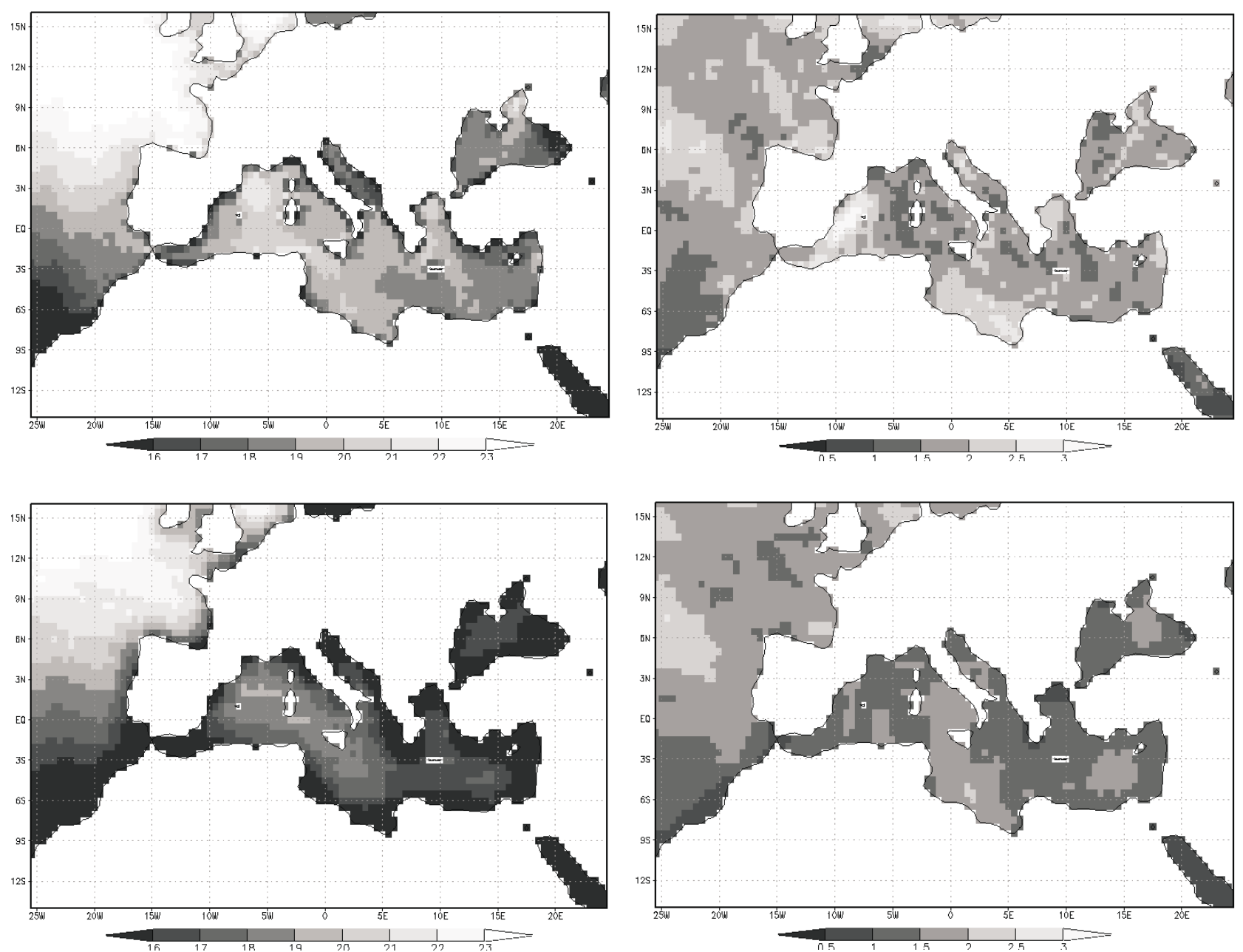

Fig. 1. Mean and standard deviation of annual-maxima wind speed for HIPOCAS (Panels (a) and (b)). The same but for NCEP (Panels (c) and (d)).

values. The first standard approach consisted in fitting the 44-years HIPOCAS annual-maxima wind speed of each offshore grid point to a Gumbel distribution, which is a particular case of the Generalized Extreme Value (GEV) distribution and has been used in several studies related to the estimation of extreme value indexes.

Before describing the results from the extreme analysis it is worth to describe the spatial distribution of annual-maxima mean values and their variability (Fig. 1). Sharp differences between hindcasted and reanalyzed data became visible, illustrating the significantly diverse characterization of wind extreme events performed by the HIPOCAS and the NCEP reanalysis. Two high-wind areas mark the spatial distribution of the HIPOCAS annual-maxima wind mean. The first one is located along the western Mediterranean, spreading from the Gulf of Lions to northern Algeria and Tunisia and the south of Sicily, whereas the second one is located in the eastern basin, affecting mainly the Aegean Sea. On the contrary, NCEP reanalysis exhibited a unique high wind nucleus that spreads along the western Mediterranean basin. With regard to the variability of the annual-maxima data,
HIPOCAS presents a maximum along the Balearic channel, as well as other areas of important variability in the Gulf of Sidra, northern Adriatic, Aegean, the Lebanon coast and in the Azov Sea. In general, NCEP reproduces lower variability than HIPOCAS and most of the aforementioned Mediterranean high variability areas derived from the hindcasted data are not found in the reanalyzed data.

Concerning wind speed values for 100 years return period of HIPOCAS data (Fig. 2a), it was remarkable that the Mediterranean spatial pattern was characterized by the presence of three maxima nuclei located south of the Gulf of Lions, the Gulf of Sidra, northern Aegean and off the Syrian coast, with levels of wind speed exceeding $30 \mathrm{~m} \mathrm{~s}^{-1}$ in the Gulf of Lions. Some other local maxima around the Strait of Gibraltar and over the northern Adriatic as well as in the Azov Sea are observed. Main features from 100 years return period patterns seemed to be driven mostly by high standard deviation areas and less by the existence of areas marked by high mean values.

On the other hand, much less defined maxima areas for 100 years return period were estimated from NCEP data, 
being non appreciable the above described HIPOCAS wind regional maxima structure. The NCEP 100 years return period case (Fig. 2b) was marked by the existence of a unique region covering the whole western Mediterranean with maximum values of the order of $26 \mathrm{~ms}^{-1}$ in an area between Corsica and Sicily and an strip towards the Balearic islands, therefore lower and generally non coincident with the HIPOCAS one. Furthermore, some areas were totally missed when compared with HIPOCAS values, as it occurred in the Strait of Gibraltar and in the northern Aegean, with differences of wind speed values reaching $12 \mathrm{~ms}^{-1}$ between both data sets (Fig. 2c). According to the obtained results, it seems clear that variability, in addition to sample mean, arises as a leading factor in the configuration of the estimated extreme wind patterns. In fact, it can be seen that main features from 100 years return period patterns are driven mostly by high standard deviation areas (more noticeable in HIPOCAS than in NCEP) and less by the existence of areas marked by high mean values.

\section{A new regional wind extreme analysis}

Additionally, a second methodology after a new statistical theory presented by Hosking and Wallis (1997) and based on a regional frequency analysis involving L-moments, was introduced to estimate more precisely the tail of the probability distribution, by gathering data from several sites instead of just one site sample. The advantage of this method relies on using information from several points instead of just one, diminishing the influence exerted by outliers to the tail of the distribution. The regional frequency analysis was applied on seven offshore areas, the first region corresponding to an Atlantic one, whereas the others were located in the Mediterranean basin. The Mediterranean regions were chosen due to the presence of prevailing regional winds, well branded and mainly linked to orographic features, as well as to cover some of the high wind Mediterranean areas. On the other hand, the Atlantic region was selected as a test zone to check the methodology over large areas with low orographic impact.

Once completed the region selection process, a measure of regional homogeneity was verified. The homogeneity of every region was evaluated by assessing whether the variations between L-moments obtained for each point samples are consistent with what is expected within a homogeneous region. Then, regional L-moments were calculated for each region and a Gumbel distribution was fitted to each regional data set as a whole, in order to estimate quantiles for 100 years return periods. Comparing the results from the standard approach methodology and the regional frequency analysis, noticeable differences appeared in the spatial distribution of the extreme winds for return periods of 100 years (Fig. 3). The spatial pattern for the Atlantic region wind speed estimations was marked by the clear existence of a latitudinal wind speed gradient, whereas the Mediterranean regions presented a slightly modified pattern. This can be interpreted as a geographical
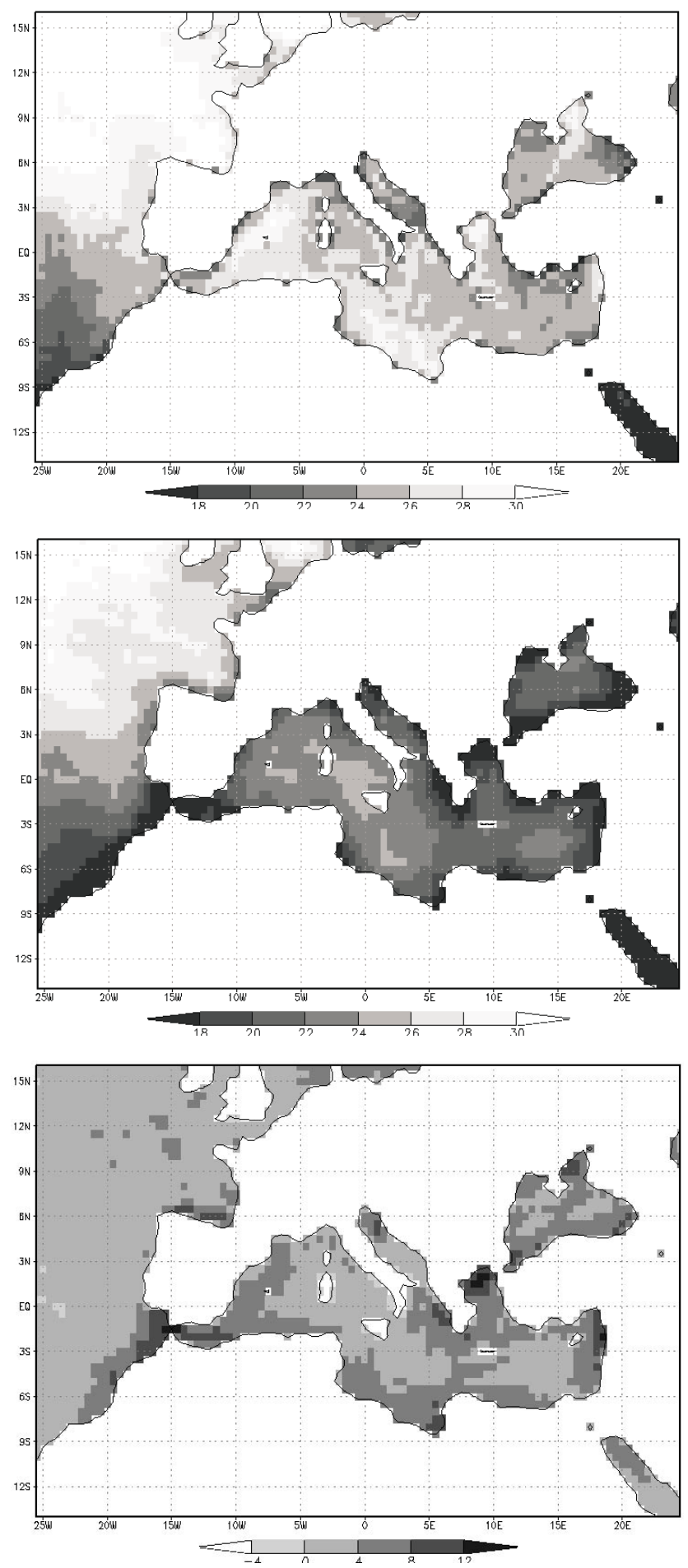

Fig. 2. (a) HIPOCAS wind speed for a 100 years return period - (b) NCEP wind speed for a 100 years return period - (c) Differences between the previous fields.

redistribution of the maxima wind speed areas within each selected region, performed by the regional frequency analysis. 

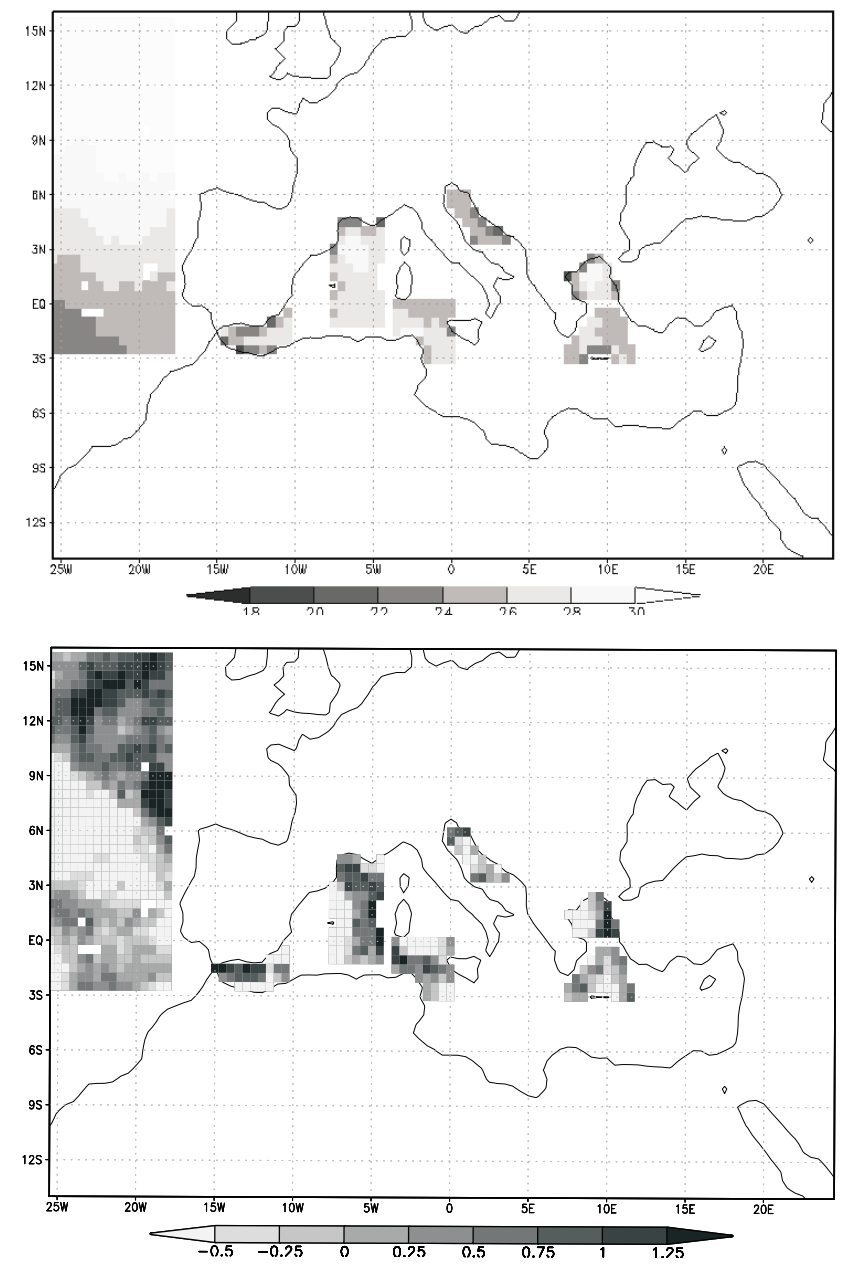

Fig. 3. (a) Wind speed winds estimated for a 100 years return period for ATLN, ALBO, LEON, SICI, BORA, EGEN and EGES regions - (b) Differences between the 100 years return period wind estimated by means of the standard approach and the regional frequency analysis.

\section{Conclusions}

Previous works have validated the HIPOCAS Mediterranean database and have proved that it reproduces extreme wind events more realistically than NCEP global reanalysis does. This paper can be seen as a continuation of this research line and presents results of statistical extreme wind analysis over the Mediterranean offshore areas performed with both databases by means of two different methods. The first one allowed obtaining expected return levels and periods through analysis of annual maxima by fitting them to a Gumbel distribution. Besides, an alternative regional statistic method based on L-moments was applied to seven regions marked by prevailing regional winds, in order to overcome the drawback of short samples available at each grid point and thus better estimate the tail of the probability distribution. The estimation of Mediterranean offshore high wind areas has been enhanced by the performed statistical analyses, enabling therefore a further better evaluation of offshore wind resources.
Edited by: V. Kotroni and K. Lagouvardos

Reviewed by: S. Lykoudis

\section{References}

Hosking, J. R. M. and Wallis, J. R.: Regional Frequency Analysis: An Approach Based on L-Moments, Cambridge University Press, Cambridge, 1997.

Kalnay, E., and co-authors: The NCEP/NCAR 40-years Reanalysis, Project. Bull. Amer. Meteorol. Soc., 77, 437-471, 1996.

Sotillo, M. G., Ratsimandresy, A. W., and Álvarez E.: Improvement of Mediterranean extreme wind characterization by means of dynamical downscaling, Proceeding of 5th Plinius Conference on Mediterranean Storms, 2003.

Sotillo, M. G., Ratsimandresy, A. W., Carretero, J. C., Bentamy, A., Valero, F., and González-Rouco, J. F.: A high-resolution 44-year atmospheric hindcast for the Mediterranean Basin: Contribution to the regional improvement of global reanalysis, Clim. Dyn., 25, 219-236, 2005. 